\title{
Building a prostate
}

Researchers have produced a functional prostate by transplanting a single stem cell expressing a characteristic set of four molecular markers into mice ${ }^{1}$. We asked three experts about the implications.

\section{Connie Eaves:}

That a single cell can regenerate a complete tissue was first demonstrated in the blood-forming system over 50 years ago. More surprising have been recent findings that highly structured epithelial tissues can also be regenerated in vivo from a single cell. Examples include mammary, skin and intestinal stem cells. Given the conservation of fundamental developmental processes, we can predict these findings will be extrapolated in the near future to many types of adult human tissue stem cells.

Director, Terry Fox Laboratory, British Columbia Cancer Agency, Vancouver, British Columbia, Canada.

\section{This work has strong implications for understanding cancer.-Massimo Loda}

\section{Massimo Loda:}

There may not be much direct clinical value in rebuilding a prostate, as it is a nonessential organ, and the required correct anatomical relationship between ducts and secretory glands in the transplant with those in the recipient would be hard to achieve. This work, however, has strong implications for understanding cancer. The findings should make it possible to define the hierarchical relationship of epithelial lineages in the prostate and to obtain a complete molecular characterization of these progenitor cells while quiescent and through differentiation. The generation of models of stem cell-derived prostate cancer will be possible through targeted genetic manipulation of these cells. Such models in turn will lead to the definition of molecularly defined subtypes of human prostate cancer-and ultimately to the understanding of the part played by prostate cancer stem cells in the development of resistance to the standard therapy, blocking signaling through the androgen receptor.

Professor of Pathology, Dana-Faber Cancer Institute, Harvard Medical School, Boston, Massachusetts, USA.

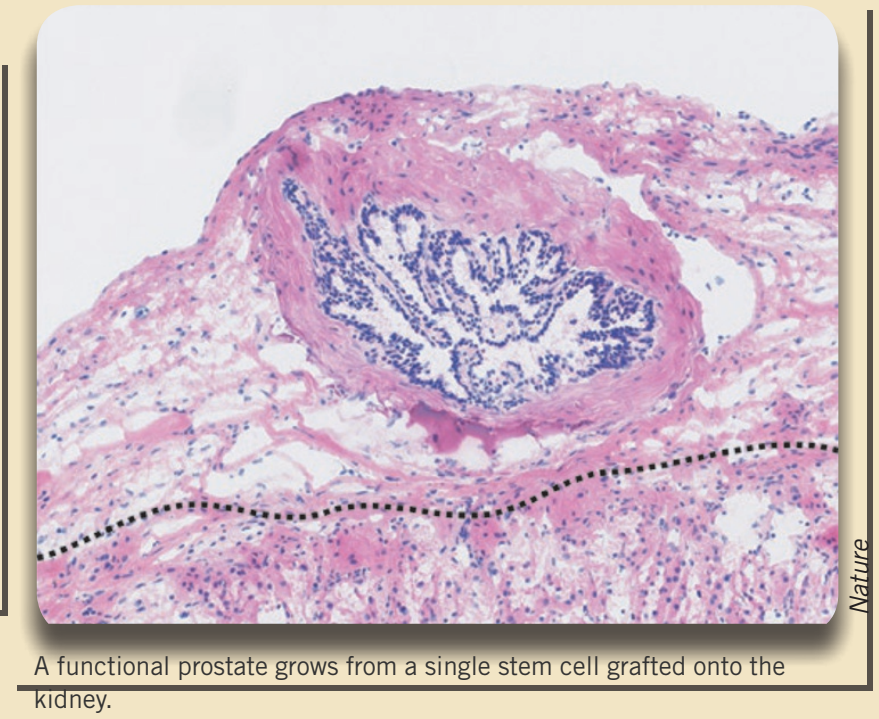

John T. Isaacs:

This work could lead to the identification of the molecular characteristics of the self-renewing cancer-initiating cell in the prostate.

Finding this cell is crucial for resolving a major therapeutic dilemma. Nonmalignant prostate epithelial adult stem cells (ASCs) do not require expression of androgen receptor for their growth or glandular development. If prostate cancer is derived from such a transformed ASC, then development of additional forms of androgen ablation therapy will be futile.

Alternatively, a growing body of data suggests that prostate cancer is derived from androgen receptor-expressing intermediate progeny of the ASC that acquire stem cell-like self-renewal ability during malignant transformation, in a process requiring androgen receptor signaling. If an intermediate cell requiring the androgen receptor is the prevalent origin of prostate cancer, then development of more effective forms of androgen ablation therapy should be a major focus of drug development.

The studies of Leong et al. should help resolve this central therapeutic question.

Professor of Oncology and Urology, Johns Hopkins School of Medicine, Baltimore, Maryland, USA.

1. Leong, K.G., Wang, B.-E., Johnson, L. \& Gao, W.Q. Generation of a prostate from a single adult stem cell. Nature published online, doi:10.1038/nature07427 (22 October 2008). 